\title{
Calidad de vida relacionada con la salud en niños con cardiopatía congénita operados durante el primer año de vida
}

\author{
Health related quality of life in children with congenital heart \\ disease that undergo cardiac surgery during their first year of life
}

\author{
Dra. Melina J. Saavedra, , Dr. Alfredo Eymann ${ }^{a}$, Dra. Lucía Péreza, \\ Dr. Julio Busanichea, Dra. Natalia Nápolib ${ }^{b}$ Dr. Pablo Marantz ${ }^{b}$ y Dr. Julián Llera ${ }^{a}$
}

\section{RESUMEN}

Introducción. En las últimas décadas, el desarrollo de métodos diagnósticos, técnicas quirúrgicas y cuidados intensivos, aumentó la sobrevida de los niños con cardiopatía congénita. En este contexto, las secuelas a largo plazo adquirieron mayor relevancia. La calidad de vida relacionada con la salud mide el impacto de las enfermedades crónicas. Nuestro objetivo fue describir la calidad de vida relacionada con la salud en pacientes con cardiopatía congénita operados durante el primer año de vida.

Población y métodos. Estudio observacional, transversal, realizado entre agosto de 2017 y diciembre de 2018 en un hospital general universitario. Se utilizó el cuestionario PedsQL 4.0, versión genérica. Se incluyeron pacientes de entre 2 y 4 años con cardiopatía congénita operados durante el primer año de vida y niños sanos. Se compararon los resultados con T-Test o Wilcoxon según la distribución observada. Se consideró significativo el valor de $\mathrm{p}<0,05$.

a. Servicio de Clínica Pediátrica, Departamento de Pediatría, Hospital Italiano de Buenos Aires, Ciudad Autónoma de Buenos Aires.

b. Servicio de Cardiología Pediátrica, Departamento de Pediatría, Hospital Italiano de Buenos Aires, Ciudad Autónoma de Buenos Aires.

Correspondencia: Dra. Melina J. Saavedra: melina.saavedra@hiba. org.ar

Financiamiento:

Ninguno.

Conflicto de intereses:

Ninguno que declarar.

Recibido: 15-4-2019

Aceptado: 21-10-2019
Cómo citar: Saavedra MJ, Eymann A, Pérez L, Busaniche J, et al. Calidad de vida relacionada con la salud en niños con cardiopatía congénita operados durante el primer año de vida. Arch Argent Pediatr 2020;118(3):166-172.

\section{INTRODUCCIÓN}

Las cardiopatías congénitas son malformaciones que se presentan en 8 de cada 1000 recién nacidos vivos. Las cardiopatías congénitas sintomáticas durante el primer año de vida tienen una incidencia de 2,5 a 3 de cada 1000 nacidos vivos; son las que exhiben mayor tasa de mortalidad y requieren corrección quirúrgica temprana. ${ }^{1}$

En las últimas décadas, como consecuencia del desarrollo de los métodos diagnósticos, como la ecografía fetal y el ecocardiograma, el diagnóstico temprano de las cardiopatías congénitas ha aumentado, lo que ha permitido el nacimiento de estos niños en instituciones con unidades neonatales de alta complejidad, servicio de cardiología y cirugía cardiovascular pediátrica o su traslado precoz a este tipo de centros. ${ }^{2-4}$

La mortalidad en el período prey posquirúrgico de estos pacientes disminuyó en forma significativa debido a los avances en las técnicas quirúrgicas y en los cuidados intensivos. El aumento de la sobrevida lleva a que algunos pacientes con estas patologías lleguen a la vida adulta. ${ }^{5}$ En este contexto, el neurodesarrollo y las secuelas a largo plazo en estos niños adquieren mayor relevancia. La principal secuela descrita en la literatura es el daño neurológico, con retraso en el desarrollo cognitivo y motor. ${ }^{5-8}$

La calidad de vida relacionada con la salud (CVRS) es un concepto que surge en la década de los noventa 
del siglo XX y describe la influencia de la salud y la enfermedad en la calidad de vida. ${ }^{9}$ Según la Organización Mundial de la Salud (OMS), la calidad de vida es la percepción que tienen los individuos de su posición en la vida en relación con sus metas, expectativas, normas e intereses, en el contexto cultural y sistema de valores en los que ellos viven. ${ }^{10}$ La CVRS se compone de características subjetivas, ya que incorpora la percepción que tiene el paciente o, en el caso de los pacientes pediátricos, de la apreciación de los padres acerca de la cotidianidad de sus hijos. Con la meta de observar este marcador de forma confiable, se desarrollaron cuestionarios que lograban medir y aportar evidencia empírica con base científica al proceso de toma de decisiones en salud. ${ }^{11}$ Sin embargo, según estudios publicados, no necesariamente la gravedad de la enfermedad y la condición clínica del paciente se asocian con su percepción de la calidad de vida. ${ }^{12}$

En la Argentina, se encuentran validados algunos instrumentos que logran objetivar la CVRS. ${ }^{13,14}$ El PedsQL es un instrumento genérico que puede ser respondido por niños sanos, pacientes con patologías crónicas o por sus padres. Permite comparar la CVRS entre pacientes sanos y enfermos, y entre distintas patologías crónicas.

La presencia de enfermedad crónica, el requerimiento de una cirugía cardiovascular a temprana edad, la necesidad de controles médicos frecuentes y de medicación diaria podrían restringir las actividades de un niño y, por lo tanto, afectar su calidad de vida. En la Argentina, no existen publicaciones que aborden exclusivamente la CVRS en los pacientes pediátricos con cardiopatía congénita. El objetivo del estudio fue describir la CVRS en los pacientes con cardiopatía congénita operados durante el primer año de vida.

\section{POBLACIÓN Y MÉTODOS}

El diseño del estudio fue observacional y analítico, de corte transversal. Se realizó entre agosto de 2017 y diciembre de 2018 en los Servicios de Cardiología Pediátrica y Clínica Pediátrica en un hospital general universitario donde se realizaban cirugías cardiovasculares en niños y que contaba con una Unidad de Terapia Intensiva Pediátrica y un área de recuperación posquirúrgica cardiovascular.

La población de niños con cardiopatía congénita fueron los pacientes operados durante el primer año de vida en el hospital que, al momento de la realización de la encuesta, tenían de 2 a 4 años. También se incluyeron niños sanos de 2 a 4 años que concurrieron a control de salud en los Consultorios Externos de Pediatría del hospital.

Los criterios de exclusión para ambos grupos fueron los síndromes genéticos y otras malformaciones congénitas mayores; prematurez menor de 30 semanas de gestación; enfermedades crónicas preexistentes, como insuficiencia renal crónica, insuficiencia hepática y enfermedades metabólicas y reumatológicas; diagnóstico de trastorno del espectro autista y la negativa a participar. En el grupo de niños con cardiopatía, se excluyeron, además, los que requirieron trasplante cardíaco, cateterismo cardíaco como método de corrección de la cardiopatía y los que se habían operado en otra institución. En la población de niños sanos, se excluyeron también los que requirieron internación por otro motivo durante el último año.

Los niños con cardiopatía se identificaron con el registro del Servicio de Cirugía Cardiovascular Pediátrica que se operaron entre diciembre de 2012 y agosto de 2015. Se seleccionaron por conveniencia según cumplieran los criterios de selección. La revisión de la historia clínica electrónica fue realizada por un becario de perfeccionamiento de cardiología pediátrica.

La población de niños sanos se seleccionó por conveniencia en relación 2 : 1 (2 pacientes sanos por cada paciente con cardiopatía) en la sala de espera de los Consultorios Externos de Pediatría del hospital. Se realizaron preguntas para identificar a los niños que cumplían con los criterios de selección. Se invitó a participar a las madres, padres o tutores acompañantes que asistieron a los controles ambulatorios de Cardiología o Pediatría o por vía telefónica. Se realizó el proceso de consentimiento informado oral a los que aceptaron participar.

\section{Instrumento de medición}

El cuestionario PedsQL 4.0, versión genérica, para niños de entre 2 y 4 años está conformado por 21 ítems agrupados en 4 dimensiones (física, emocional, social, escolar) y puntajes sumarios (total, física y psicosocial). Se contesta siguiendo una escala de tipo Likert $(0=$ nunca fue un problema; 1 = casi nunca fue un problema; 2 = algunas veces fue un problema; 3 = frecuentemente fue un problema; $4=$ casi siempre fue un problema). El cuestionario es autoadministrado y fue respondido por uno de los padres o tutores. Las respuestas fueron 
transformadas a una escala de 0 a 100. Por lo tanto, los puntajes más altos se correspondían con mejor CVRS.

En ambos grupos, se analizaron las respuestas con el objetivo de describir con qué frecuencia se reportaron problemas relevantes (frecuentemente o casi siempre fue un problema) en cada esfera. Asimismo, se recolectaron los siguientes datos: sexo del niño; edad al momento de la cirugía; edad al momento de la encuesta; máximo nivel educativo de los padres; padres y hermanos convivientes; procedencia de la familia; sexo de quien respondía la encuesta; diagnóstico de cardiopatía, número de cirugías cardiovasculares durante el primer año de vida; complicaciones quirúrgicas y uso de medicación cardiológica al momento de la encuesta. El estudio fue aprobado por el Comité de Ética de Protocolos de Investigación de nuestra Institución (Protocolo número IRB00010193).

\section{Cálculo de tamaño muestral y análisis estadístico}

Para el cálculo del tamaño muestral, se consideró el estudio de validación del cuestionario genérico PedsQL, versión argentina, en español. ${ }^{13}$ Se tomó la media del total del cuestionario de calidad de vida en el grupo de niños cardiópatas de 2 a 4 años de 74 puntos (desvío estándar -DE-13) y la media en el grupo de niños sanos de igual edad de 82 puntos (DE 12). El cálculo fue de 30 niños con cardiopatía y de 60 niños sanos para un intervalo de confianza del $95 \%$ y un poder del $80 \%$ para un test a dos colas con una relación $1: 2$.

Las variables categóricas se expresaron en número absoluto y frecuencia, y las variables continuas, en media y DE o mediana y rango intercuartílico (RIC) según la distribución observada. Para el análisis estadístico, se realizó la comparación entre niños sanos y con cirugía cardiovascular mediante el test de Wilcoxon dada la distribución no paramétrica de los datos. Se utilizó el software Stata 13.

\section{RESULTADOS}

Se realizó la encuesta a 31 niños con cardiopatía congénita y a 62 niños sanos que cumplían con los criterios de selección (véase el diagrama de flujo en la Figura 1). Las características demográficas de ambos grupos se muestran en la Tabla 1. La mediana de edad de los niños fue de 3,3 años (RIC: 2,8-4,2) y el $76 \%$ de las encuestas fueron respondidas por las madres. El $54 \%$ de los padres tenía un nivel educativo

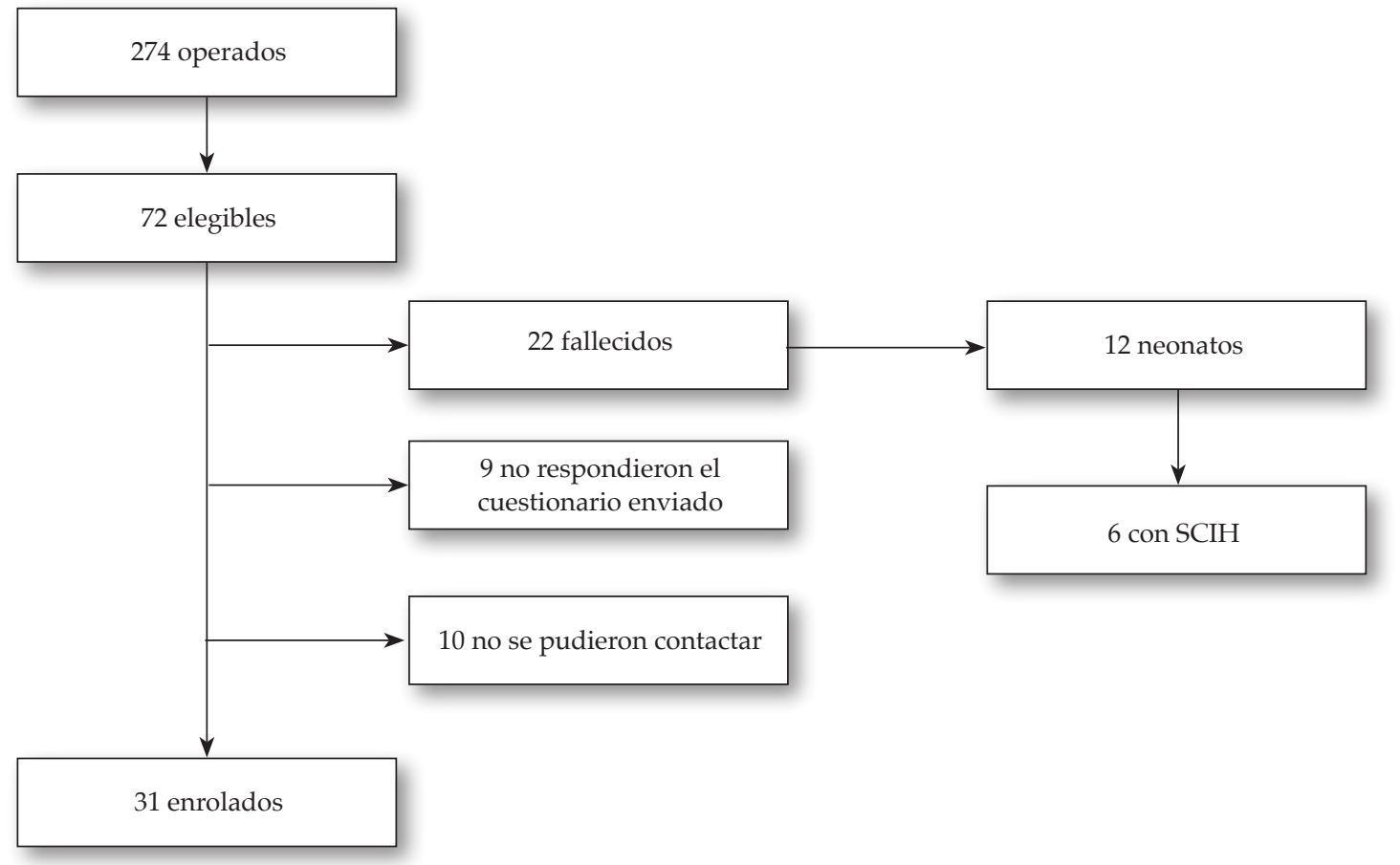

SCIH: síndrome de corazón izquierdo hipoplásico. 
alto con estudios universitarios finalizados. El $93 \%$ de los padres eran convivientes y, en su mayoría, tenían un hijo (el $51 \%$ ) o dos (el $42 \%$ ). El $95 \%$ de los niños sanos asistía al jardín de infantes, mientras que, en el grupo de niños con cardiopatía, concurría un $80 \%$.

El $61 \%$ de los niños con cardiopatía eran de sexo masculino; el $51 \%$ provenían de la Ciudad Autónoma de Buenos Aires (CABA) y Gran Buenos Aires, y el resto eran de localidades más alejadas. Los diagnósticos de los niños con cardiopatías congénitas se muestran en la Tabla 2. El $26 \%$ tenían fisiología de ventrículo único, de los cuales el $50 \%$ se encontraba, al momento de la realización de la encuesta, en estadio Fontan.

En ese momento, el seguimiento cardiológico ambulatorio tenía una media de 41,4 meses y el 61,3\% de los pacientes tenía prescrita medicación cardiovascular. La primera cirugía fue en el período neonatal en un $61,3 \%$. En cuanto al número de cirugías al momento de la encuesta, el $51,3 \%$ presentó una, y el $29 \%$, tres. El porcentaje de pacientes con complicaciones posquirúrgicas fue variable dependiendo del número de cirugía. Nueve pacientes (el $29 \%$ ) presentaron complicaciones en más de una de ellas.

Nuestro estudio muestra que no se encontró diferencia $(p=0,10)$ entre la CVRS global de la población con cardiopatía congénita y de los niños sanos. Sin embargo, se observaron puntajes más bajos con diferencia estadísticamente significativa en las esferas social $(p=0,0092)$ y escolar ( $\mathrm{p}=0,0001)$ (Tabla 3$)$.

Las respuestas del PedsQL muestran que el $29 \%$ de los padres de pacientes con cardiopatía reportaron problemas relevantes (frecuentemente o casi siempre fue un problema) en, al menos, un aspecto (funcionamiento físico en el $6,4 \%$, funcionamiento emocional en el $19,3 \%$, funcionamiento social en el 9,6\% y funcionamiento escolar en el 6,4\%). Además, el $17,7 \%$ de los padres de los niños sanos mostraron estos resultados (funcionamiento físico en el $9,6 \%$, funcionamiento emocional en el 9,6\%, funcionamiento social en el 1,6\%, funcionamiento escolar en el $0 \%$ ).

Tabla 1. Características demográficas de los niños sanos y con cardiopatía congénita

\begin{tabular}{|c|c|c|c|}
\hline & Niños sanos $(n=62)$ & Niños con cardiopatía congénita $(\mathrm{n}=31)$ & Valor de $p$ \\
\hline \multicolumn{4}{|l|}{ Sexo, n (\%) } \\
\hline Varón & $32(51,6)$ & $19(61,3)$ & \multirow[t]{2}{*}{0,37} \\
\hline Mujer & $30(48,4)$ & $12(38,7)$ & \\
\hline Edad, mediana (RIC) & $3(2,58-4)$ & $3,83(3-4,41)$ & 0,04 \\
\hline \multicolumn{4}{|l|}{ Localidad, $\mathrm{n}(\%)$} \\
\hline Ciudad Autónoma de Buenos Aires & $56(90,3)$ & $6(19,4)$ & \multirow[t]{5}{*}{$<0,001$} \\
\hline Gran Buenos Aires & $6(9,6)$ & $10(32,3)$ & \\
\hline Menor de 400 km & - & $3(9,7)$ & \\
\hline Entre 400 y $1000 \mathrm{~km}$ & - & $6(19,4)$ & \\
\hline Más de $1000 \mathrm{~km}$ & - & $6(19,4)$ & \\
\hline \multicolumn{4}{|l|}{ Sexo del padre que responde, $\mathrm{n}(\%)$} \\
\hline Varón & $10(16,1)$ & $12(38,7)$ & \multirow[t]{2}{*}{0,016} \\
\hline Mujer & $52(83,9)$ & $19(61,3)$ & \\
\hline \multicolumn{4}{|l|}{ Máximo nivel educativo alcanzado, n (\%) } \\
\hline Universitario & $37(59,7)$ & $14(45,5)$ & \multirow[t]{4}{*}{0,6} \\
\hline Terciario & $12(19,4)$ & $9(29)$ & \\
\hline Secundario & $12(19,4)$ & $8(25,8)$ & \\
\hline Primario & $1(1,6)$ & $0(0)$ & \\
\hline \multicolumn{4}{|l|}{ Conviven los padres, $\mathrm{n}(\%)$} \\
\hline & $60(96,8)$ & $27(87,1)$ & 0,07 \\
\hline \multicolumn{4}{|l|}{ Hermanos convivientes, $\mathrm{n}(\%)$} \\
\hline 0 & $26(41,9)$ & $11(35,5)$ & \multirow[t]{4}{*}{0,43} \\
\hline 1 & $26(41,9)$ & $13(41,9)$ & \\
\hline 2 & $7(11,3)$ & $5(16,1)$ & \\
\hline 3 o más & $3(4,8)$ & $2(6,4)$ & \\
\hline \multicolumn{4}{|l|}{ Concurrencia a jardín de infantes, $\mathrm{n}(\%)$} \\
\hline & $59(95,1)$ & $25(80,6)$ & 0,02 \\
\hline
\end{tabular}

RIC: rango intercuartílico. 
TABLA 2. Características de los pacientes con cardiopatía congénita $(n=31)$

\begin{tabular}{lc}
\hline Diagnóstico & $\mathbf{n}(\%)$ \\
\hline CIV & $3(9,6)$ \\
Coartación de aorta & $2(6,45)$ \\
CIV + coartación de aorta & $2(6,45)$ \\
Estenosis aórtica crítica & $1(3,2)$ \\
Complejo de Shone & $2(6,45)$ \\
Canal auriculoventricular & $1(3,2)$ \\
d-TGV simple (d-TGV) & $2(6,45)$ \\
TGV con CIV & $2(6,45)$ \\
Tronco arterioso & $1(3,2)$ \\
Tetralogía de Fallot & $2(6,45)$ \\
DSVD** & $3(9,6)$ \\
Atresia pulmonar con CIV & $2(6,45)$ \\
Ventrículo único* & $8(26)$ \\
Medicación cardiológica, $n(\%)$ & $19(61,3)$ \\
Edad en meses en la primera cirugía, $n(\%)$ & \\
De 0 a 1 & $19(61,3)$ \\
De 1 a 3 & $6(19,3)$ \\
De 4 a 6 & $5(16)$ \\
De 7 a 9 & $1(3,3)$ \\
De 9 a 12 & - \\
Número de cirugías, $n(\%)$ & \\
1 & \\
2 & $16(51,6)$ \\
3 & $6(19,3)$ \\
Días totales de ARM, $n(\%)$ & $9(29)$ \\
De 1 a 5 días & \\
De 5 a 10 días & $16(51,5)$ \\
De 10 a 15 días & $7(22,5)$ \\
Más de 15 días & $4(13)$ \\
Complicaciones, $n(\%)$ & $4(13)$ \\
Primera cirugía (n = 31) & \\
Segunda cirugía (n $=17)$ & $16(51)$ \\
Tercera cirugía (n = 8) & $6(35)$ \\
\hline & $4(50)$ \\
\hline
\end{tabular}

* SCIH; atresia tricuspídea tipo 1B; doble entrada ventricular + atresia tricuspídea + atresia pulmonar; doble entrada ventricular + transposición de grandes vasos + estenosis pulmonar; atresia pulmonar con septum integro.

** DSVD tipo Fallot; DSVD + coartación de aorta; DSVD + TGV + CIV.

CIV: comunicación interventricular; TGV: transposición de los grandes vasos; DSVD: doble salida del ventrículo derecho; ARM: asistencia respiratoria mecánica.

\section{DISCUSIÓN}

Sobre la base de los reportes de los padres, nuestro estudio muestra que no se encontró diferencia estadísticamente significativa $(p=0,10)$ entre la CVRS global de la población de 2 a 4 años con cardiopatía congénita operados durante el primer año de vida y la de los niños sanos de la misma edad. Resultados similares se obtuvieron en los aspectos físico $(\mathrm{p}=0,88)$, psicosocial $(0,43)$ y emocional $(0,66)$. Sin embargo, se observaron puntajes más bajos con diferencia estadísticamente significativa en las esferas social $(p=0,0092)$ y escolar $(p=0,0001)$. El objetivo del presente estudio fue describir la CVRS en pacientes de 2 a 4 años con cardiopatía congénita operados durante el primer año de vida utilizando una herramienta validada, el PedsQL 4.0, versión genérica.

La CVRS es un marcador fundamental que mide el impacto de las enfermedades crónicas. ${ }^{9}$ En este contexto, están emergiendo estudios que abordan esta temática a nivel mundial. Los resultados son variables y se encuentran reportes que concluyen que estos pacientes presentan puntajes menores en los cuestionarios de calidad de vida comparados con pacientes con otras enfermedades crónicas y con niños sanos. ${ }^{5-8}$

Además, existen trabajos publicados en los que no se encontraron diferencias significativas entre la CVRS de los pacientes con cardiopatía y de los niños sanos. ${ }^{15-19}$ La mayoría involucran a pacientes con diagnóstico de transposición de los grandes vasos (TGV). En la publicación de Brosig et al., ${ }^{19}$ utilizaron el cuestionario PedsQL en una cohorte similar a la nuestra, compuesta por 26 pacientes con diagnósticos de síndrome de corazón izquierdo hipoplásico (SCIH) y TGV, y no se encontraron diferencias significativas en los aspectos físico, emocional y social. La mayoría de los niños no concurría al jardín de infantes, por lo tanto, no reportaron tanto el puntaje escolar como el total.

Los resultados de nuestro estudio,

TABLA 3. Calidad de vida relacionada con la salud en niños con cardiopatía congénita y en controles sanos

\begin{tabular}{lccc}
\hline & Sanos $(\mathbf{n}=\mathbf{6 2})$ & Cardiopatía congénita $(\mathbf{n}=\mathbf{3 1})$ & Valor de $p$ \\
\hline Total & $92,5(87,5-96,25)$ & $89,7(75,8-95,4)$ & 0,10 \\
Físico & $96,87(90,62-100)$ & $93,75(87,5-100)$ & 0,88 \\
Psicosocial & $90(85-95)$ & $87,5(80-95)$ & 0,43 \\
Emocional & $82,5(75-90)$ & $85(75-100)$ & 0,66 \\
Social & $100(90-100)$ & $90(85-100)$ & 0,0092 \\
Escolar & $100(91,66-100)$ & $83,3(66,6-91,6)$ & 0,0001 \\
\hline
\end{tabular}

Valores expresados en mediana y rango intercuartílico. 
probablemente, se deban a múltiples factores. Por un lado, los puntajes similares en las dos poblaciones en los dominios físico y emocional podrían deberse a la influencia de la familia en su desarrollo. Por otro lado, los puntajes significativamente menores en las esferas social y escolar podrían ser consecuencia de la interacción del niño con la sociedad por fuera del ámbito familiar. Otro factor que podría influir es la escolarización, ya que fue menor la cantidad de niños con cardiopatía que concurría al jardín de infantes $(p=0,02)$ y el ausentismo escolar debido a enfermedad o a controles médicos programados fue mucho más frecuente en este grupo. La cantidad de días de ausentismo se ve incrementada si el paciente vive alejado del hospital, y el $80 \%$ de esta población vivía por fuera de la CABA $(p<0,001)$.

En algunas cohortes, se observó que el medio socioeconómico tenía influencia en la CVRS y que las madres reportaron puntajes menores comparadas con los padres encuestados. ${ }^{20-24} \mathrm{En}$ nuestro estudio, hubo una proporción mayor de padres de niños con cardiopatía congénita que respondieron el cuestionario comparado con los padres de niños sanos $(p=0,016)$. Ambos grupos presentaron alto nivel educativo $(p=0,6) y$, en su mayoría, eran convivientes $(p=0,07)$.

En la Argentina, se ha descrito la CVRS en niños con distintas enfermedades crónicas, como asma, enfermedad celíaca, trasplante hepático y de médula ósea, enfermedad pulmonar obstructiva crónica (EPOC), virus de la inmunodeficiencia humana (VIH), cáncer, insuficiencia renal crónica terminal y cardiopatía congénita. Se observaron, en la mayoría de estos pacientes, puntajes significativamente más bajos que los controles sanos en todos los dominios. ${ }^{13,25}$ Estos estudios fueron realizados, en su mayoría, en el ámbito de la salud pública. En comparación con la validación argentina del cuestionario PedsQL, los puntajes de nuestros pacientes fueron superiores. La media en nuestra población con cardiopatía congénita fue de 86,32 versus 74,61 en la validación y la media en niños sanos fue 91,42 versus $82,19 .{ }^{13}$ Es probable que los resultados sean diferentes, dado que nuestro estudio se realizó en un hospital privado de la comunidad, en una población con medio socioeconómico mayor e incluyó a una población de 2 a 4 años.

El estudio presenta limitaciones por tener un pequeño número de pacientes y por haber sido realizado en un solo centro. Asimismo, las poblaciones de niños comparadas tenían distinta procedencia y nivel de escolaridad. Se utilizó un instrumento que medía la CVRS sobre la base de la opinión de los padres de los niños. Se ha visto, en algunos estudios, que esta puede diferir de la realidad que perciben los pacientes. ${ }^{26}$ Además, podría haber sesgo de selección teniendo en cuenta que los padres que eligieron no responder el formulario podrían haber estado influenciados por una percepción negativa de la calidad de vida de sus hijos.

La CVRS es un indicador médico emergente. Dado el aumento de la sobrevida de los pacientes con cardiopatía congénita, el interés por poder estimar la CVRS comienza a formar parte del plan de cuidados a largo plazo de estos niños. Los resultados de este estudio ponderan la importancia de la evaluación de la calidad de vida en el seguimiento de los pacientes con enfermedades crónicas. Sin embargo, deben ser confirmados por estudios multicéntricos con mayor número de pacientes.

\section{CONCLUSIÓN}

Nuestra cohorte de pacientes preescolares con cardiopatía congénita operados durante el primer año de vida tiene una calidad de vida global comparable con la que llevan los niños sanos, excepto en las escalas correspondientes a funcionamiento social y escolar.

\section{Agradecimientos}

A las doctoras Clara Ceriani y Julia Saulino por su colaboración.

\section{REFERENCIAS}

1. Hoffman JI, Kaplan S. The incidence of congenital heart disease. J Am Coll Cardiol. 2002; 39(12):1890-900.

2. Chock V, Lee HC. Neurodevelopmental outcomes for infants born with congenital heart disease. Neoreviews. 2014; 15(8):e344-53.

3. Govindaswami B, Jegatheesan P, Song D. Oxygen saturation screening for critical congenital heart disease. Neoreviews. 2012; 13(12):e724-31.

4. Guerchicoff M, MrantzP, Infante J, Villa A, etal. Evaluación del impacto del diagnóstico precoz de las cardiopatías congénitas. Arch Argent Pediatr. 2004; 102(6):445-50.

5. Australian Institute of Health and Welfare. Heart, Stroke \& Vascular Diseases: Australian Facts 2004. AIHW No. CVD 27. Canberra: AIHW and National Heart Foundation of Australia; 2004. (Cardiovascular Disease Series No. 22).

6. Mahle WT, Clancy RR, Moss EM, Gerdes M, et al. Neurodevelopmental outcome and lifestyle assessment in school-aged and adolescent children with hypoplastic left heart syndrome. Pediatrics. 2000; 105(5);1082-9.

7. Snookes SH, Gunn JK, Eldridge BJ, Donath SM, et al. A systematic review of motor and cognitive outcomes after early surgery for congenital heart disease. Pediatrics. 2010; 125(4):e818-27. 
8. Gaynor JW, Stopp C, Wypij D, Andropoulos DB, et al. Neurodevelopmental outcomes after cardiac surgery in infancy. Pediatrics. 2015; 135(5):816-25.

9. Guyatt GH, Feeny DH, Patrick D. Measuring health-related quality of life. Ann Intern Med. 1993; 118(8): 622-9.

10. World Health Organization. WHOQOL: MeasuringQuality of life. Geneva: WHOQOL Group: 1997.

11. Testa MA, Simonson DC. Assessment of quality-of-life outcomes. N Engl J Med. 1996; 334(13):835-40.

12. Mussatto K, Tweddell J. Quality of life following surgery for congenital cardiac malformations in neonates and infants. Cardiol Young. 2005; 15(Suppl 1):174-8.

13. Roizen M, Rodríguez S, Bauer G, Medin G, et al. Initial validation of the Argentinean Spanish version of the PedsQLTM 4.0 Generic Core Scales in children and adolescents with chronic diseases: acceptability and comprehensibility in low-income settings. Health Qual Life Outcomes. 2008; 6:59.

14. Grupo de trabajo sobre Calidad de Vida en la Infancia. Calidad de vida relacionada con la salud en pediatría. Instrumentos utilizados para su evaluación. Arch Argent Pediatr. 2014; 112(6):571-6.

15. Culbert EL, Ashburn DA, Cullen-Dean G, Joseph JA, et al. Quality of life of children after repair of transposition of the great arteries. Circulation. 2003; 108(7):857-62.

16. Møyen Laane K, Meberg A, Otterstad JE, Frøland G, et al. Quality of life in children with congenital heart defects. Acta Paediatr. 1997; 86(9):975-80.

17. Dunbar-Masterson C, WypijD, BellingerDC, RappaportLA, etal. General health status of children with D-transposition of the great arteries after arterial switch operation. Circulation. 2001; 104(12 Suppl 1): I138-42.
18. Ekman-Joelsson BM, Berntsson L, Sunnergårdh J. Quality of life in children with pulmonary atresia and intact ventricular septum. Cardiol Young. 2004; 14(6):615-21.

19. Brosig CL, Mussatto KA, Kuhn EM, Tweddell JS. Psychosocial outcomes for preschool children and families after surgery for complex congenital heart disease. Pediatr Cardiol. 2007; 28(4):255-62.

20. Hovels-Gurich H, Konrad K, Wiesner M, Minkenberg R, et al. Long term behavioral outcome after neonatal arterial switch operation for transposition of the great arteries. Arch Dis Child. 2002; 87(6):506-10.

21. Lawoko S, Soares JJ. Quality of life among parents of children with congenital heart disease, parents of children with other diseases and parents of healthy children. Qual Life Res. 2003; 12(6):655-66.

22. Latal B, Helfricht S, Fischer JE, Bauersfeld U, et al. Psychological adjustment and quality of life in children and adolescents following open-heart surgery for congenital heart disease: a systematic review. BMC Pediatr. 2009; 9:6.

23. Roizen M, Figueroa C, Salvia L, Comité de Calidad de Vida y Salud. Calidad de vida relacionada con la salud en niños con enfermedades crónicas: comparación de la visión de los niños, sus padres y sus médicos. Arch Argent Pediatr. 2007; 105(4):305-13.

24. Garcia Guerra G, Robertson CM, Alton GY, Joffe AR, et al. Quality of life 4 years after complex heart surgery in infancy. J Thorac Cardiovasc Surg. 2013; 145(2):482-8.e2.

25. Sánchez C, Eymann A, De Cunto C, D'Agostino D. Quality of life in pediatric liver transplantation in a single-center in South America. Pediatr Transplant. 2010; 14(3):332-6.

26. Patel BJ, Lai L, Goldfield G, Sananes R, et al. Psychosocial health and quality of life among children with cardiac diagnoses: agreement and discrepancies between parent and child reports. Cardiol Young. 2017; 27(4):713-21. 